\title{
Índice de Comportamentos de Risco: construção e análise das propriedades psicométricas
}

\author{
Risky Behavior Index: Construction and \\ analysis of psychometric properties
}

\author{
Cássia Ferrazza ALVES \\ Jana Gonçalves ZAPPE ${ }^{1}$ \\ Débora Dalbosco DELL'AGLIO'
}

\begin{abstract}
Resumo
Adolescentes adotam comportamentos de risco que podem favorecer ou prejudicar seu desenvolvimento, o que demanda atenção científica e profissional. Este estudo apresenta o processo de construção e análise das propriedades psicométricas do Índice de Comportamento de Risco. O processo envolveu quatro etapas: revisão da literatura, seleção dos itens, análise preliminar do instrumento, análise final das propriedades psicométricas. Foi utilizado um banco de dados com informações de 466 estudantes de escolas públicas de Porto Alegre, no estado do Rio Grande do Sul, com idade entre 11 e 19 anos. A versão final do Índice possui 17 itens, distribuídos em quatro fatores: comportamento sexual de risco, uso de substâncias, comportamento infracional e comportamento suicida. O instrumento apresentou consistência interna satisfatória $(\alpha=0,84)$, assim como permitiu avaliar simultaneamente um conjunto de comportamentos de risco, bem como identificar a prevalência e coocorrência desses comportamentos em adolescentes ou jovens adultos, contribuindo para o desenvolvimento científico e profissional de atenção à adolescência.
\end{abstract}

Palavras-chave: Adolescentes; Comportamento de risco; Psicometria.

\begin{abstract}
Adolescents adopt risk taking behaviours that may contribute or hinder their development, which demand scientific and professional attention. The aim of the present study is to discuss the construction process and the analysis of the psychometric properties of the Behavioral Risk Index. The process included four steps: literature review, item selection, preliminary and final analysis of psychometric properties. A database with 466 students, between the ages of 11-19 years, from public schools in Porto Alegre, Rio Grande do Sul, was used. The final version of the index has 17 items, divided into four factors (risky sexual behavior, substance use, delinquent and suicidal behavior) and it presented satisfactory internal consistency $(\alpha=0.84)$. This instrument enables the simultaneous evaluation of multiple behavioral risks and identifies the prevalence and co-occurrence of these behaviors in adolescents or young adults, contributing to the scientific and professional attention to adolescence.
\end{abstract}

Keywords: Adolescents; Risk behavior; Psychometrics.

\footnotetext{
1 Universidade Federal do Rio Grande do Sul, Instituto de Psicologia, Programa de Pós-Graduação em Psicologia. R. Ramiro Barcellos, 2600, Sala 115, Santa Cecília, 90035-003, Porto Alegre, RS, Brasil. Correspondência para/Correspondence to: D.D. DELL'ALGIO. E-mail: <debora.dellaglio@ufrgs.br>
}

7.7 
A adolescência compreende um conjunto de transformações biopsicossociais que se processam entre a infância e a idade adulta e culminam com a conquista de um papel na sociedade. Apesar de haver uma tendência em caracterizar a adolescência como um momento de dificuldades, conflitos e alterações de humor, cada vez mais tem sido enfatizada a necessidade de considerar que esse também é um momento de intensa exploração e descoberta de múltiplas oportunidades (Senna \& Dessen, 2012).

No contexto da busca de novas experiências e da exploração de novos ambientes, situações e companhias, os adolescentes podem se engajar em inúmeros comportamentos de risco, os quais podem ser importantes para o desenvolvimento da autonomia e da capacidade de fazer escolhas, mas também podem acarretar consequências negativas a curto, médio ou longo prazo. Esses comportamentos podem abranger uso de álcool, cigarro e drogas, conduta antissocial, comportamento sexual de risco, comportamento suicida, comportamento alimentar de risco, prática inadequada de atividades físicas, comportamento de risco no trânsito, dificuldades escolares, entre outros (Guedes \& Lopes, 2010; Huang, Lanza, Murphy, \& Hser, 2012).

Diversos estudos têm buscado compreender os fatores associados à adoção de comportamentos de risco na adolescência, investigando sua prevalência, a coocorrência e os fatores de risco e proteção (Farias Júnior et al., 2009; Hidalgo, Piedra, Díaz, \& González, 2012; Huang et al., 2012; Moller \& Haustein, 2014). Por exemplo, no estudo de Farias Júnior et al. (2009) com jovens brasileiros, os comportamentos mais prevalentes foram relacionados à alimentação não saudável, comportamento sexual de risco e nível insuficiente de atividade física, e mais da metade dos adolescentes envolveram-se em mais de dois comportamentos de risco simultaneamente. A coocorrência dos comportamentos de risco também foi analisada por Huang et al. (2012), sendo que os adolescentes com maior uso do álcool ou maconha apresentaram maior probabilidade em ter iniciação sexual precoce e comportamento infrator. Moller e Haustein (2014) indicaram que o envolvimento em comportamentos de risco no trânsito, como o excesso de velocidade, relaciona-se com a proximidade de amigos com comportamento semelhante e à consequente aceitação por parte deles.

Em termos de fatores de proteção, estudos da área de epidemiologia cognitiva demonstraram que sujeitos com mais habilidades cognitivas tendem a adotar comportamentos mais saudáveis, pois fumam menos, não bebem excessivamente, evitam acidentes, exercitam-se mais e mantêm uma dieta saudável (Deary, 2013; Deary \& Batty, 2007). Em termos de fatores de risco, Steinberg (2004) destaca que, durante a adolescência, há o desenvolvimento gradual do sistema de autorregulação (responsável pelo controle dos impulsos, planejamento e previsão de riscos) e um aumento do interesse por novidades e busca de sensações, tornando o jovem mais sensível a estímulos emocionais e mais propenso a tomar decisões de risco.

Assim, cabe diferenciar entre atitude de risco e comportamento de risco. Os estudos envolvendo atitude de risco geralmente abordam aspectos como tomada de decisão de risco e influência de aspectos neurológicos no processo de decisão (Smith, Chein, \& Steinberg, 2013). Por outro lado, os estudos envolvendo comportamento de risco abordam a manifestação propriamente dita de comportamentos potencialmente prejudiciais.

A maioria dos estudos que abordam diversos grupos de comportamentos de risco salienta que eles se inter-relacionam de forma complexa e possuem muitos antecedentes comuns e, por isso, é recomendado o estudo de diversos comportamentos de risco conjuntamente (Dryfoos, 1990; Guedes \& Lopes, 2010; Huang et al., 2012). No Brasil, a maioria dos estudos investiga comportamentos isolados, e não há um programa de vigilância que monitore o conjunto de comportamentos de risco que afetam a saúde e o desenvolvimento da população jovem (Guedes \& Lopes, 2010). Além disso, os estudos utilizam diferentes métodos e instrumentos, dificultando a unificação dos resultados para uma compreensão mais abrangente do problema.

Partindo destas lacunas, elaborou-se um Índice de Comportamentos de Risco (ICR) que abran- 
ge quatro áreas - comportamento sexual de risco, envolvimento em atos infracionais, uso de substâncias, comportamento suicida -, com o objetivo de avaliar o comportamento de risco em adolescentes brasileiros, de forma integrada. O ICR foi desenvolvido a partir de uma ampla revisão de estudos sobre o tema e de um banco de dados de pesquisa com o Questionário da Juventude Brasileira - versão II (QJB) (Dell'Aglio, Koller, Cerqueira-Santos, \& Colaço, 2011). Este artigo tem por objetivo, portanto, apresentar o processo de elaboração do ICR em sua versão final, bem como suas principais propriedades psicométricas.

\section{Método}

\section{Participantes}

Os dados deste estudo procedem do banco de dados da pesquisa "Adolescentes em diferentes contextos: família e institucionalização" (Dell'Aglio, 2012), que buscou identificar fatores de risco e proteção no desenvolvimento de jovens.

Naquele estudo, foi utilizada uma amostra aleatória, por conglomerados, a partir do sorteio de escolas de Ensino Fundamental e Médio pertencentes à rede pública da cidade de Porto Alegre (RS). O número de participantes foi estabelecido através de cálculo amostral (Barbetta, 2001), com margem de erro de 4,0\%. Na amostra final, participaram 466 adolescentes com idade entre 11 e 19 anos (Média - $M=14,99$; Desvio-Padrão - DP = 1,57), conforme a definição estabelecida pela Organização Mundial da Saúde, que compreende a adolescência como o período entre 10 e 19 anos de idade (World Health Organization [WHO], 2004). Dentre os participantes, 63,3\% pertenciam ao sexo feminino. Os participantes responderam a todas as questões selecionadas para compor o ICR. A aplicação do instrumento foi coletiva, em sala de aula, com duração média de 60 minutos.

\section{Instrumentos}

Este estudo apresenta o desenvolvimento do instrumento ICR, composto a partir de 17 itens, dis- tribuídos em quatro fatores: comportamento sexual de risco, uso de substâncias, comportamento infracional e comportamento suicida. O processo de construção do instrumento e as análises de suas propriedades psicométricas são apresentados nos resultados.

\section{Procedimentos}

A construção do Índice seguiu as etapas sugeridas por DeVellis (2012):

- A primeira etapa consistiu em uma revisão sistemática sobre comportamentos de risco em bancos de dados nacionais e internacionais, sendo considerados principalmente os critérios utilizados para avaliação de comportamentos de risco.

- Na segunda etapa, foram selecionados os itens que compõem o ICR, a partir das questões contidas no QJB (Dell'Aglio et al., 2011). Esse instrumento é um questionário amplo, que investiga fatores de risco e proteção em adolescentes, com 77 questões relacionadas a família, saúde, educação, rede de apoio e comportamentos de risco, entre outros. Foram selecionadas apenas as questões que investigam quatro tipos de comportamento de risco: comportamento sexual, uso de substâncias, envolvimento em atos infracionais e comportamento suicida, pontuando-se cada item a partir dos critérios identificados na literatura.

- A terceira etapa consistiu em uma análise preliminar das propriedades psicométricas da primeira versão do ICR. Alguns itens e pontuações foram revistos, dando origem à versão final.

A análise da versão final configurou a quarta e última etapa de construção do instrumento. A avaliação das propriedades psicométricas do ICR incluiu uma análise fatorial exploratória e a avaliação da confiabilidade através do alfa de Cronbach.

A pesquisa foi aprovada pelo Comitê de Ética em Pesquisa com Seres Humanos, do Instituto de Psicologia da Universidade Federal do Rio Grande do Sul (Protocolo n²009060, aprovado em 07/01/2010), tendo seguido todas as recomendações para pesquisas com seres humanos. 


\section{Resultados e Discussão}

\section{Primeira parte: definição dos itens do ICR}

A partir das questões disponíveis no QJB, foram selecionados quatro grupos de itens, que representam as áreas mais estudadas nas pesquisas sobre comportamento de risco na adolescência: comportamento sexual de risco, uso de substâncias, comportamento infracional e comportamento suicida.

\section{Comportamento sexual de risco}

Os estudos revisados apontam que os principais comportamentos sexuais de risco adotados por adolescentes são o não uso de preservativos ou contraceptivos (Farias Júnior et al., 2009; George, Alary, \& Otis, 2007), a idade precoce de iniciação sexual (Bachanas et al., 2003; Bassols, Boni, \& Pechansky, 2010) e o relacionamento com múltiplos parceiros (Bassols et al., 2010; Cruzeiro et al., 2010; Sena \& Colares, 2008).

Com relação à idade da iniciação sexual, alguns autores consideram como um comportamento de risco a ocorrência da primeira relação antes dos 12 anos (Bassols et al., 2010), 13 anos (Brener et al., 2002; Linetzky, Morello, Virgolini, \& Ferrante, 2011), 14 anos (Huang et al., 2012), ou antes dos 15 anos (Campos-Arias, Ceballo, \& Herazo, 2010; Crockett, Raffaelli, \& Shen, 2006; Raffaelli \& Crockett, 2003). A idade precoce do primeiro intercurso sexual pode ser considerada como um comportamento de risco tanto em função da imaturidade emocional do adolescente para administrar relacionamentos mais íntimos como em função da maior vulnerabilidade do adolescente para adotar outros comportamentos de risco (Huang et al., 2012).

O número de parceiros sexuais é um aspecto relacionado a comportamento de risco, podendo-se considerar o número de parceiros de toda a vida sexual do jovem (Kosunen, Kaltiala-Heino, Rimpelä, \& Laippala, 2003; Raffaelli \& Crockett, 2003) ou no
Zimmerman, \& Caldwell, 2007; Roche, Ahmed, \& Blum, 2008). De modo geral, os estudos consideram que, quanto maior o número de parceiros, maior o risco (Fergus et al., 2007; Kosunen et al., 2003). Crockett et al. (2006) utilizaram uma escala de risco que variou de zero a quatro, considerando que ter dois ou mais parceiros sexuais no ano é um indicador de alto risco. Kosunen et al. (2003) avaliaram o número de parceiros sexuais durante a vida do jovem e consideraram como alto risco ter mais de cinco parceiros.

Quanto aos métodos de proteção contra gravidez e Doenças Sexualmente Transmissíveis (DST), o não uso de preservativo ou o uso inconsistente são considerados como comportamentos de risco. O uso consistente é definido como uso contínuo em todas as relações sexuais, enquanto o uso inconsistente se refere ao não uso e ao uso irregular ou raro de preservativo (Bertoni et al., 2009; Busseri, Willoughby, Chalmers, \& Bogaert, 2008; George, Alary, \& Otis, 2007; Hair, Park, Ling, \& Moore, 2009a; Raffaelli \& Crockett, 2003).

No que tange ao uso de métodos contraceptivos, há estudos que investigaram o uso ou não uso de tais métodos na última relação sexual (Caminis, Henrich, Ruchkin, Schwab-Stone, \& Martin, 2007; Kosunen et al., 2003), com o último parceiro sexual (Graaf, Vanwesenbeeck, Meijer, Woertman, \& Meeu, 2009) ou na primeira relação sexual (Jones, Darroch, \& Singh, 2005). Autores como Jones et al. (2005) compreendem que o não uso de métodos contraceptivos já constitui risco ao jovem, e autores como Kosunen et al. (2003) investigaram o risco conforme o tipo de método, considerando o seu grau de eficácia (contraceptivos orais, coito interrompido e tabela do ciclo menstrual).

A partir dos estudos citados, foram selecionadas quatro questões do QJB, para avaliar comportamento sexual de risco. O primeiro item avalia a idade da iniciação sexual (questão 42c do QJB), considerando que, quanto menor a idade, maior a pontuação. O segundo item avalia o número de parceiros fixos e não fixos no último ano, considerando que, quanto maior o número de parceiros, maior a pontuação. A frequência do uso de preservativo no último ano (questão 45 do QJB) constitui 
o terceiro item do ICR, sendo que, quanto menos frequente for o uso, maior é a pontuação. O quarto item se refere ao uso de métodos contraceptivos, considerando que, quanto mais eficaz o contraceptivo utilizado, menor a pontuação (questão 55 em que o adolescente indicava o método utilizado).

\section{Uso de substâncias (álcool, cigarro e drogas)}

O uso experimental ou continuado de substâncias pode trazer prejuízos ao desenvolvimento do jovem, sendo considerado um comportamento de risco para a saúde. Existem autores que diferenciam entre o não uso, o uso experimental e o uso contínuo (Malta et al., 2010), enquanto outros autores diferenciam apenas entre o uso e o não uso (Bertoni et al., 2009; Crockett et al., 2006; Kogan et al., 2010).

No Índice de Comportamento de Risco, optou-se por diferenciar a experimentação do uso frequente de substâncias, pois se compreende que experimentar tais substâncias expõe o jovem a um risco menor do que o consumo frequente. No QJB, a experimentação foi investigada na questão 34 . Para o ICR, foi considerado que o jovem que nunca experimentou não apresenta risco; que aquele que experimentou álcool ou tabaco apresenta risco baixo; e que aquele que experimentou outras drogas (ilícitas) apresenta risco alto. Embora toda substância seja ilícita para adolescentes no Brasil (Brasil, 1990), essa distinção leva em consideração o fato de que, quando o adolescente experimenta uma droga caracterizada como ilícita, expõe-se a riscos paralelos, como o envolvimento com o tráfico e a criminalidade. Assim, o quinto item do ICR avalia a experimentação de álcool, cigarro e drogas, considerando maior a pontuação para a experimentação de drogas ilícitas.

Quanto ao consumo de substâncias, a idade de iniciação tem sido considerada nos estudos (Burrone et al., 2010; Madu \& Matla, 2003), pois o início precoce é um importante preditor para o uso abusivo (Acosta, Fernández, \& Pillon, 2011; McCarty et al., 2004). Os estudos investigam a idade de iniciação a partir de intervalos como, por exemplo, entre 12 e 14 anos; 15 e 17 anos; 18 e 20 anos (Hidalgo et al., 2012), ou somente questionando a idade de início (Brener et al., 2002; Madu \& Matla, 2003; Miller, Strathdee, Kerr, Li, \& Wood, 2006; Oliveira, Martins, Reato, \& Akerman, 2010). No QJB, a questão 34 questionou a idade de início do consumo de álcool, cigarro e drogas, a qual deu origem aos itens 6, 7 e 8 do ICR, atribuindo maior pontuação a idades mais precoces.

Além da idade de iniciação no consumo de substâncias, os estudos investigam também a frequência do uso (Beebe et al., 2008; Farias Junior, Mendes, \& Barbosa, 2007; Farias Júnior et al., 2009; Kogan et al., 2010; Linetzky, Morello, Virgdini, \& Ferrante, 2011). Roche et al. (2008) verificaram a quantidade do uso de álcool por meio de uma escala que variou de nunca a diariamente. Com relação ao uso do cigarro, os estudos avaliam a frequência de uso no mês (Hair et al., 2009a), na semana (Farias Júnior et al., 2009), ou no dia (Busseri et al., 2008). A frequência do uso de drogas ou outras substâncias tem sido pesquisada considerando o uso mensal (Beebe et al., 2008; Huang et al., 2012; Kokkevi, Richardson, Florescu, Kuzmanf, \& Stergar, 2007), o qual pode variar de consumo leve a consumo pesado (Kokkevi et al., 2007; Tavares, Béria, \& Lima, 2001).

No Questionário da Juventude Brasileira, a questão 36 investigou a frequência do uso de álcool, cigarro e drogas, verificando o consumo no último ano e no último mês. O consumo no último ano foi averiguado por meio de uma questão dicotômica (sim/não). Quanto ao consumo no último mês, foi investigado o uso de ao menos uma vez na semana, uso de uma a quatro vezes na semana, e uso de mais de cinco vezes na semana. Dessa forma, a frequência do uso de álcool, cigarro ou drogas configurou os itens 9, 10 e 11 do ICR, considerando que, quanto maior a frequência do uso, mais alta a pontuação.

Diversos estudos indicam que frequentemente o consumo de drogas se dá em um contexto de interações sociais do adolescente com amigos e 
familiares, uma vez que a percepção acerca das normas de pares e familiares sobre o uso de drogas é um aspecto relacionado com o consumo de drogas (Moral, Rodríguez, \& Ovejero, 2010). Nesse sentido, ter amigos e familiares usuários de drogas é considerado um fator de risco para o uso de substâncias na adolescência (Kokkevi et al., 2007; Raphaelli, Azevedo, \& Hallal, 2011). Além disso, quando o uso de drogas se dá fora de um contexto de interação social, quando o adolescente se encontra sozinho, é considerado um risco mais alto. Assim, o item 12 do ICR investiga com quem o adolescente consome substâncias (a partir da questão 37 do QJB), considerando que o uso solitário configura risco mais alto que o uso com pares e familiares. Dessa forma, oito itens compuseram o fator uso de substâncias do ICR, contemplando os aspectos teóricos investigados.

\section{Envolvimento em atos infracionais}

Com relação ao envolvimento em atos infracionais, dois aspectos têm sido investigados: o tipo e a quantidade de atos infracionais cometidos. Com relação ao tipo, alguns estudos diferenciam entre atos sem violência, como fugas noturnas, furto em lojas, fazer corridas de carro e atos com violência, como envolvimento em brigas, porte de armas e participação em gangue (Busseri et al., 2008; Caminis et al., 2007; Farias Júnior et al., 2009; Hair et al., 2009a; Kokkevi et al., 2007). Ao pesquisar atos infracionais, muitos autores consideram o envolvimento no último ano (Busseri et al., 2008; Farias Júnior et al., 2009; Hair et al., 2009a). Com relação à quantidade de atos infracionais cometidos, os estudos utilizam escalas que variam de "nunca" a "cinco vezes ou mais" ou "sempre", considerando que a maior frequência de envolvimento configura risco mais alto (Auerbach \& Gardiner, 2012; Huang et al., 2012; Kokkevi et al., 2007; Ritakallio, Kaltiala-Heino, Kivivuori, \& Rimpela, 2005).

A partir desses estudos, foram elaborados dois itens para avaliar comportamento infracional a partir da questão 64 do QJB. O item 13 avalia o tipo de ato infracional cometido, considerando-se que destruição de propriedade, assalto, roubo e venda de drogas são envolvimentos mais graves do que brigas e pichações. O item 14 avalia o número de situações ilegais em que o jovem se envolveu, considerando que, quanto maior seu número, maior a pontuação.

\section{Comportamento suicida}

O comportamento suicida tem sido investigado como comportamento de risco ao desenvolvimento do adolescente, incluindo tanto a ideação quanto a tentativa de suicídio (Nkansah-Amankra et al., 2012). Ao avaliar os dois comportamentos juntos, Nkansah-Amankra et al. (2012) classificaram os participantes em três categorias: 'nenhuma história de comportamento suicida', 'ideação suicida, mas não comportamento suicida' e 'tentativa de suicídio'.

No Questionário da Juventude Brasileira, as questões 66a e 67a investigaram o comportamento suicida por meio de uma questão com resposta dicotômica (sim/não) para ideação suicida, e outra para tentativa de suicídio. Dessa forma, ideação e tentativa de suicídio configuram o item 15 do ICR, compreendendo que o jovem que tentou suicídio encontra-se em maior risco do que aquele que apenas pensou nisso.

Além da presença de ideação ou tentativa de suicídio, os estudos verificam a frequência desses eventos, utilizando escalas que variam de zero a cinco vezes (Nkansah-Amankra et al., 2012; Zhang, Wang, Xia, Liu, \& Jung, 2012) ou zero a três vezes ou mais (Boeninger, Masyn, Feldman, \& Conger, 2010; Cukrowicz et al., 2011), considerando diferentes intervalos de tempo, em geral, no último mês ou ano. No QJB, as questões 66b e 67b pesquisaram o número de ideações e tentativas de suicídio, respectivamente. Assim, a frequência de ideação suicida foi atribuída no item 16 do ICR, e a frequência de tentativas de suicídio no item 17 , considerando que, quanto maior a frequência, maior a pontuação.

Após a etapa de definição dos itens do ICR para cada um dos tipos de comportamentos de risco estudados, foram atribuídas as pontuações para 
cada item. Inicialmente, foram atribuídos valores que variaram de zero a três, compreendendo zero como inexistência de comportamento de risco, um como risco baixo, dois como risco médio e três como risco alto, e então se procedeu à análise preliminar.

\section{Segunda parte: análise preliminar, revisão e elaboração da versão final}

A análise da primeira versão do ICR, com cada item podendo variar de zero a três, resultou em alfa de Cronbach de 0,77, o que é um indicador de boa consistência interna. Porém, observou-se que a pontuação atribuída foi muito restritiva na avaliação dos comportamentos de risco, o que ocasionou uma diferenciação muito pequena entre os participantes. Por exemplo, ao avaliar uso de preservativo, o jovem que fazia uso contínuo (sempre ou quase sempre) pontuava zero, aquele que fez uso em algumas vezes pontuava um, quem usou poucas vezes pontuava dois, e quem nunca usou pontuava três. Porém, muitos estudos consideram que, quando o jovem deixa de usar preservativo apenas uma vez, ele já adotou um comportamento de risco, de forma que a pontuação estabelecida desviou-se daquelas atribuídas em outros estudos.

Partindo disso, adotou-se uma nova pontuação que variou de zero a dois, sendo que zero significa ausência de risco, um significa risco baixo, e dois significa risco alto. Dessa forma, chegou-se à versão final do ICR, que inclui 17 itens que variam de zero a dois pontos, com uma pontuação total de zero a 34 pontos, conforme pode ser observado na Tabela 1.

\section{Terceira parte: descrição das qualidades psicométricas do ICR}

As análises indicaram que a pontuação total dos participantes variou de zero a 23 pontos, com média de 5,71 ( $D P=5,23)$. O alfa de Cronbach obtido na versão final do ICR foi de 0,84 , indicando alta consistência interna.

Para analisar as relações entre os itens da escala, buscando identificar se os itens agrupados nos quatro tipos de comportamentos de risco formam fatores, realizou-se uma análise fatorial exploratória. Inicialmente verificou-se a adequação da matriz correlacional quanto aos pressupostos necessários à análise multivariada, como a ausência de multicolinearidade e a fatorabilidade dos dados. $\mathrm{O}$ indicador Kaiser Meyer Olken (KMO) foi igual a 0,67, e o teste de esfericidade de Bartlett foi significativo $(p<0,001)$. Com base na significância conceitual dos itens, decidiu-se pela extração de quatro fatores pelo método de componentes principais. Esse método possibilita a redução dos dados, buscando o mínimo de fatores necessários que expliquem a porção máxima da variância total (Hair, Black, Babin, Anderson, \& Tathan, 2009b). A análise da matriz de componentes principais revelou que os itens referentes a comportamento sexual de risco apresentaram carga cruzada, carregando simultaneamente em dois fatores. Os itens relacionados com uso de substâncias, comportamento infracional e comportamento suicida carregaram em três fatores diferentes, com cargas fatorais significantes. Para eliminar as cargas cruzadas, foi realizado o método de rotação Varimax, o qual se concentra na simplificação das colunas da matriz fatorial, fornecendo uma separação mais clara dos fatores (Hair, 2009b). A Tabela 2 apresenta a matriz de cargas fatoriais comuns rotacionada por Varimax.

Analisando os resultados apresentados, pode-se constatar que os quatro fatores identificados, a partir da matriz de cargas fatoriais, correspondem às quatro áreas de comportamentos de risco incluídas no ICR: comportamento sexual de risco, uso de substâncias, comportamento infracional e comportamento suicida, e que todos os itens possuem cargas significativas. Dessa forma, conclui-se que o ICR é um instrumento adequado para avaliar comportamentos de risco na adolescência, considerando um conjunto de fatores de forma integrada.

Logo, com a construção do ICR, buscou-se obter um instrumento que permitisse avaliar um conjunto de comportamentos de risco que podem ser adotados por adolescentes. A análise fatorial permitiu identificar os fatores estabelecidos a priori 
Tabela 1

Itens e pontuações do Índice de Comportamentos de Risco

\begin{tabular}{|c|c|c|c|}
\hline Item & Questão & Pontuação & \\
\hline 1 & Idade na primeira relação sexual & $\begin{array}{l}\text { Não teve relação sexual ou teve a partir de } 17 \text { anos } \\
14-16 \text { anos } \\
\text { Até } 13 \text { anos }\end{array}$ & $\begin{array}{l}0 \\
1 \\
2\end{array}$ \\
\hline 2 & Número de parceiros sexuais no último ano & $\begin{array}{l}\text { Nenhum } \\
\text { Até } 2 \text { parceiros } \\
3 \text { parceiros ou mais }\end{array}$ & $\begin{array}{l}0 \\
1 \\
2\end{array}$ \\
\hline 3 & Frequência de uso de camisinha no último ano & $\begin{array}{l}\text { Sempre } \\
\text { Nem sempre } \\
\text { Nunca }\end{array}$ & $\begin{array}{l}0 \\
1 \\
2\end{array}$ \\
\hline 4 & Uso de métodos contraceptivos & $\begin{array}{l}\text { Eficaz } \\
\text { Ineficaz } \\
\text { Nenhum }\end{array}$ & $\begin{array}{l}0 \\
1 \\
2\end{array}$ \\
\hline 5 & Experimentação de álcool, cigarro ou drogas & $\begin{array}{l}\text { Nunca experimentou } \\
\text { Experimentou álcool ou cigarro } \\
\text { Experimentou droga ilícita }\end{array}$ & $\begin{array}{l}0 \\
1 \\
2\end{array}$ \\
\hline 6 & Idade na primeira vez que usou álcool & $\begin{array}{l}\text { A partir de } 18 \text { anos } \\
\text { De } 15 \text { a } 17 \text { anos } \\
\text { Até } 14 \text { anos }\end{array}$ & $\begin{array}{l}0 \\
1 \\
2\end{array}$ \\
\hline 7 & Idade na primeira vez que usou cigarro & $\begin{array}{l}\text { A partir de } 18 \text { anos } \\
\text { De } 15 \text { a } 17 \text { anos } \\
\text { Até } 14 \text { anos }\end{array}$ & $\begin{array}{l}0 \\
1 \\
2\end{array}$ \\
\hline 8 & $\begin{array}{l}\text { Idade na primeira vez em que usou droga ou outras } \\
\text { substâncias }\end{array}$ & $\begin{array}{l}\text { Nunca usou } \\
15 \text { anos ou mais } \\
\text { Até } 14 \text { anos }\end{array}$ & $\begin{array}{l}0 \\
1 \\
2\end{array}$ \\
\hline 9 & Frequência de uso de álcool & $\begin{array}{l}\text { Não usou no último mês } \\
1 \text { vez na semana } \\
\text { Mais de } 1 \text { vez na semana }\end{array}$ & $\begin{array}{l}0 \\
1 \\
2\end{array}$ \\
\hline 10 & Frequência de uso de cigarro & $\begin{array}{l}\text { Não usou no último mês } \\
1 \text { vez na semana } \\
\text { Mais de } 1 \text { vez na semana }\end{array}$ & $\begin{array}{l}0 \\
1 \\
2\end{array}$ \\
\hline 11 & Frequência de uso de drogas ou outras substâncias & $\begin{array}{l}\text { Não usou no último mês } \\
1 \text { vez na semana } \\
\text { Mais de } 1 \text { vez na semana }\end{array}$ & $\begin{array}{l}0 \\
1 \\
2\end{array}$ \\
\hline 12 & Parceiro em consumo de drogas & $\begin{array}{l}\text { Não usa drogas } \\
\text { Amigos/namorado(a) ou Família } \\
\text { Sozinho }\end{array}$ & $\begin{array}{l}0 \\
1 \\
2\end{array}$ \\
\hline 13 & Envolvimento em situações ilegais & $\begin{array}{l}\text { Não } \\
\text { Brigas e agressão física/violência contra pessoas e envolvimento em } \\
\text { pichação } \\
\text { Destruição de propriedade, assalto, roubo ou venda de drogas }\end{array}$ & $\begin{array}{l}0 \\
1\end{array}$ \\
\hline 14 & Número de situações ilegais em que se envolveu & $\begin{array}{l}\text { Nenhuma } \\
\text { Uma } \\
\text { Duas ou mais }\end{array}$ & $\begin{array}{l}0 \\
1 \\
2\end{array}$ \\
\hline 15 & Ideação e tentativa de suicídio & $\begin{array}{l}\text { Nunca pensou ou tentou } \\
\text { Já pensou, mas nunca tentou } \\
\text { Já tentou se matar }\end{array}$ & $\begin{array}{l}0 \\
1 \\
2\end{array}$ \\
\hline 16 & Frequência de ideações suicidas & $\begin{array}{l}\text { Nunca pensou } \\
\text { Uma vez } \\
\text { Duas ou mais vezes }\end{array}$ & $\begin{array}{l}0 \\
1 \\
2\end{array}$ \\
\hline 17 & Frequência de tentativas de suicídio & $\begin{array}{l}\text { Nunca tentou } \\
\text { Uma vez } \\
\text { Duas ou mais vezes }\end{array}$ & $\begin{array}{l}0 \\
1 \\
2\end{array}$ \\
\hline
\end{tabular}




\begin{tabular}{|c|c|c|c|c|c|}
\hline \multirow{2}{*}{ Itens } & \multicolumn{4}{|c|}{ Fator } & \multirow{2}{*}{ Comunalidade } \\
\hline & 1 & 2 & 3 & 4 & \\
\hline Idade na primeira relação sexual & & & 0,84 & & 0,79 \\
\hline Número de parceiros sexuais no último ano & & & 0,78 & & 0,75 \\
\hline Frequência de uso de camisinha & & & 0,69 & & 0,56 \\
\hline Uso de métodos contraceptivos & & & 0,63 & & 0,43 \\
\hline Experimentou álcool, cigarro ou drogas & 0,80 & & & & 0,75 \\
\hline Idade na primeira vez que usou álcool & 0,61 & & & & 0,43 \\
\hline Idade na primeira vez que usou cigarro & 0,65 & & & & 0,49 \\
\hline Idade na primeira vez que usou droga ilícita & 0,64 & & & & 0,49 \\
\hline Frequência de uso de álcool & 0,57 & & & & 0,36 \\
\hline Frequência de uso de cigarro & 0,64 & & & & 0,45 \\
\hline Frequência de uso de drogas ilícitas & 0,51 & & & & 0,26 \\
\hline Parceiro em consumo de drogas & 0,71 & & & & 0,53 \\
\hline Envolvimento em situações ilegais & & & & 0,93 & 0,92 \\
\hline Número de situações ilegais & & & & 0,94 & 0,94 \\
\hline Comportamento suicida & & 0,96 & & & 0,95 \\
\hline Número de vezes que pensou em se matar & & 0,88 & & & 0,80 \\
\hline Número de vezes que tentou se matar & & 0,80 & & & 0,66 \\
\hline
\end{tabular}

(comportamento sexual de risco, uso de substâncias, comportamento infracional e comportamento suicida). A partir desse índice, será possível identificar a prevalência, a coocorrência e os fatores de risco e proteção associados a comportamentos de risco em adolescentes, contribuindo para o avanço do conhecimento nessa área. Futuros estudos que utilizem o ICR poderão contribuir para o conhecimento da validade e da fidedignidade do mesmo.

Considerando-se que a adoção de comportamentos de risco pode tanto favorecer quanto prejudicar o desenvolvimento, torna-se muito relevante que futuros estudos informem acerca dos aspectos que podem diferenciar comportamentos favoráveis e desfavoráveis, visando a oferta de subsídios a pais e educadores para o monitoramento e supervisão do desenvolvimento durante a adolescência. Além disso, o conhecimento sobre fatores de risco e proteção associados pode levar ao desenvolvimento de estratégias de intervenção que visem minimizar os riscos e promover proteção.

Adotando-se os resultados de estudos da área de epidemiologia cognitiva, entende-se que a promoção de habilidades cognitivas pode constituir uma estratégia de proteção ao desenvolvimento, uma vez que há associação entre habilidades cogni- tivas e adoção de comportamentos mais saudáveis (Deary, 2013; Deary \& Batty, 2007). Assim, sugere-se a realização de estudos com a população brasileira que busquem conhecer a relação entre habilidades cognitivas, como a inteligência, e o envolvimento em comportamentos de risco.

Contudo, algumas limitações desta pesquisa devem ser destacadas. Tendo em vista que o Índice foi criado a partir de questões retiradas de outro instrumento, os tipos de comportamentos de risco avaliados não permitem esgotar a ampla variedade de comportamentos que podem ser adotados por adolescentes. Assim, sugere-se que estudos futuros avancem na qualificação do ICR, com a inclusão de outros tipos de comportamentos, aumentando as possibilidades de investigação da prevalência e da coocorrência dos mesmos.

A amostra utilizada para analisar as propriedades psicométricas do ICR foi composta por adolescentes; porém, considera-se relevante investigar também jovens adultos. Além disso, são necessários estudos longitudinais, que avaliem as trajetórias de desenvolvimento do engajamento em comportamentos de risco durante a adolescência até a idade adulta. Estudos com adolescentes que vivem em diferentes contextos, expostos a situações de 
risco diferenciadas, também podem contribuir para uma maior compreensão das relações entre a manifestação desses comportamentos e as variáveis ambientais. Enfim, destaca-se que um maior conhecimento sobre o comportamento de risco na adolescência pode trazer contribuições às políticas públicas direcionadas aos jovens, que favoreçam ações de prevenção e intervenção para a promoção do desenvolvimento saudável.

\section{Referências}

Acosta, L., Fernández, A., \& Pillon, S. (2011). Factores sociales para el uso de alcohol en adolescentes y jóvenes. Revista Latino-Americana de Enfermagem, 19(Spe), 771-81.

Auerbach, R., \& Gardiner, C. (2012). Moving beyond the trait conceptualization of self-esteem: The prospective effect of impulsiveness, coping, and risky behavior engagement. Behaviour Research and Therapy, 50(10), 596-603.

Bachanas, P. J., Morris M. K., Lewis-Gess J. K., SarettCuasay E. J., Flores A. L., Sirl, K. S., \& Sawyer, M. K. (2003). Psychological adjustment, substance use, HIV knowledge, and risky sexual behavior in at-risk minority females: Developmental differences during adolescence. Journal of Pediatric Psychology, 27(4), 373-384.

Barbetta, P. A. (2001). Estatística aplicada às ciências sociais. Florianópolis: UFSC.

Bassols, A. M., Boni, R., \& Pechansky, F. (2010). Alcohol, drugs, and risky sexual behavior are related to HIV infection in female adolescents. Revista Brasileira de Psiquiatria, 32(4), 361-368.

Bertoni, N., Bastos, F. I., Mello, M.B., Makuch, M. Y., Sousa, M. H., Osis, M. J., \& Faúndes, A. (2009). Uso de álcool e drogas e sua influência sobre as práticas sexuais de adolescentes de Minas Gerais, Brasil. Cadernos de Saúde Pública, 25(6), 1350-1360.

Beebe, L., Vesely, S., Oman, R., Tolma, E., Aspy, C., \& Rodine, S. (2008). Protective assets for non-use of alcohol, tobacco and other drugs among urban American Indian youth in Oklahoma. Maternal Child Health Journal, 12(Supl 1), 582-590.

Boeninger, D., Masyn, K., Feldman, B., \& Conger, R. (2010). Sex differences in developmental trends of suicide ideation, plans, and attempts among European American adolescents. Suicide and Life-Threatening Behavior, 40(5), 451-464.

Brasil. Presidência da República. (1990). Lei n 8.069/1990, de 13 de julho de 1990. Dispõe sobre o Estatuto da Criança e do Adolescente e dá outras providências.
Brener, N., Kann, L., McManus, T., Kinchen, S., Sundberg, E., \& Ross, J. (2002). Reliability of the 1999 youth risk behavior survey questionnaire. Journal of Adolescent Health, 31(4), 336-342.

Burrone, M. S., Bueno, S. M. V., Costa Jr, M. L., Enders, J., Fernández, R. A., \& Vasters, G. P. (2010). Análisis de la frecuencia de experimentación y consumo de drogas de alumnos de escuelas de nivel medio. Revista Latino-Americana de Enfermagem, 18(Spe), 648-654.

Busseri, M. A., Willoughby, T., Chalmers, H., \& Bogaert, A. F. (2008). On the association between sexual attraction and adolescent risk behavior involvement: Examining mediation and moderation. Developmental Psychology, 44(1), 69-80.

Caminis, A., Henrich, C., Ruchkin, V., Schwab-Stone, M., \& Martin, A. (2007). Psychosocial predictors of sexual initiation and high-risk sexual behaviors in early adolescence. Child and Adolescent Psychiatry and Mental Health, 1(14), 1-12.

Campos-Arias, A., Ceballo, C., \& Herazo, E. (2010). Prevalence of pattern of risky behaviors for reproductive and sexual health among middle-and high-school students. Revista Latino-Americana de Enfermagem, 18(2), 170-174.

Crockett, L., Raffaelli, M., \& Shen, Y. (2006). Linking selfregulation and risk proneness to risky sexual behavior: Pathways through peer pressure and early substance use. Journal of Research on Adolescence, 16(4), 503-525.

Cruzeiro, A. L. S., Souza, L. D. M., Silva, R. A., Pinheiro, R. T., Rocha, C. L. A., \& Horta, B. L. (2010). Comportamento sexual de risco: fatores associados ao número de parceiros sexuais e ao uso de preservativo em adolescentes. Ciência \& Saúde Coletiva, 15(Supl 1), 1149-1158.

Cukrowicz, K., Schlegel, E., Smith, P., Jacobs, M., Orden, K., Paukert, A., ... Joiner, T. (2011). Suicide ideation among college students evidencing subclinical depression. Journal of American College Health, 59(7), 575-581.

Deary, I. J. (2013). Intelligence. Current Biology, 23(16), 673-676.

Deary, I. J., \& Batty, G. D. (2007). Cognitive epidemiology. Journal of Epidemiology Community Health, 61(5), 378-384.

Dell'Aglio, D. D. (2012). Adolescentes em diferentes contextos: família e institucionalização. Relatório de Pesquisa (Conselho Nacional de Desenvolvimento Científico e Tecnológico, No 507433/2010-6). Porto Alegre: Universidade Federal do Rio Grande do Sul.

Dell'Aglio, D. D., Koller, S. H., Cerqueira-Santos, E., \& Colaço, V. (2011). Revisando o Questionário da Juventude Brasileira: uma nova proposta. In D. D. Dell'Aglio \& S. H. Koller (Eds.), Adolescência e juventude: vulnerabilidade e contextos de proteção (pp.259-270). São Paulo: Casa do Psicólogo. 
DeVellis, R. (2012). Scale development (3 ${ }^{\text {rd }}$ ed.). Los Angeles: Sage.

Dryfoos, J. (1990). Adolescents at risk: Prevalence and prevention. New York: Oxford University.

Farias Júnior, J., Mendes, J., \& Barbosa, D. (2007). Associação entre comportamentos de risco à saúde em adolescentes. Revista Brasileira de Cineantropometria \& Desempenho Humano, 9(3), 250-256.

Farias Júnior, J. C., Nahas, M. V., Barros, M. V., Loch, M. R., Oliveira, E. S., De Bem, M. F., \& Lopes, A. S. (2009). Comportamentos de risco à saúde em adolescentes no Sul do Brasil: prevalência e fatores associados. Revista Panamericana de Salud Pública, 25(4), 344-352.

Fergus, S., Zimmerman, M. A., \& Caldwell, C. H. (2007). Growth trajectories of sexual risk behavior in adolescence and young adulthood. American Journal of Public Health, 97(6), 1096-1101.

George, C., Alary, M., \& Otis, J. (2007). Correlates of sexual activity and inconsistent condom use among high-school girls in Dominica. West Indian Medicine Journal, 56(5), 433-438.

Graaf, H.,Vanwesenbeeck, I., Meijer, S., Woertman, L., \& Meeu, W. (2009). Sexual trajectories during adolescence: Relation to demographic characteristics and sexual risk. Archive Sexual Behaviour, 38(2), 276-282.

Guedes, D. P., \& Lopes, C. C. (2010). Validação da versão brasileira do Youth Risk Behavior Survey 2007. Revista de Saúde Pública, 44(5), 840-850.

Hair, E. C., Park, M. J., Ling, T., J., \& Moore, K. A. (2009a). Risky behaviors in late adolescence: Co-occurrence, predictors, and consequences. Journal of Adolescent Health, 45(3), 253-261.

Hair, J., Black, W., Babin, B., Anderson, R., \& Tathan, R. (2009b). Análise multivariada dos dados (6 $6^{\text {rd }} \mathrm{ed}$.). Porto Alegre: Bookman.

Hidalgo, J., Piedra, J., Díaz, K., \& González, M. (2012). Hábito de fumar en la adolescência. Revista Cubana de Medicina General Integral, 28(3), 282-289.

Huang, D., Lanza, I., Murphy, D., \& Hser, Y. (2012). Parallel development of risk behaviors in adolescence: Potential pathways to co-occurrence. International Journal of Behavioral Development, 36(4), 247-257.

Jones, R., Darroch, J., \& Singh, S. (2005). Religious differentials in the sexual and reproductive behaviors of young women in the United States. Journal of Adolescent Health, 36(4), 279-288.

Kogan, S. M., Beach, S. R., Philibert, R. A., Brody, G. H., Chen, Y. F., \& Lei, M. K. (2010). 5-HTTLPR status moderates the effect of early adolescent substance use on risky sexual behavior. Health Psychology, 29(5), 471-476.

Kokkevi, A., Richardson, C., Florescu, S., Kuzmanf, M., \& Stergar, E. (2007). Psychosocial correlates of substance use in adolescence: A cross-national study in six
European countries. Drug and Alcohol Dependence, 86(1), 67-74.

Kosunen, E., Kaltiala-Heino, R., Rimpelä, M., \& Laippala, P. (2003). Risk-taking sexual behaviour and selfreported depression in middle adolescence: A schoolbased survey. Child: Care, Health \& Development, 29(5), 337-344.

Linetzky, B., Morello, P., Virgolini, M., \& Ferrante, D. (2011). Resultados de la primera encuesta nacional de salud escolar: Argentina, 2007. Archivos Argentinos de Pediatría, 109(2), 111-116.

Madu, S., \& Matla, M., (2003). Illicit drug use, cigarette smoking and alcohol drinking behaviour among a sample of high school adolescents in the Pietersburg area of the Northern Province, South Africa. Journal of Adolescence, 26(1), 121-136.

Malta, D., Sardinha, L., Mendes, I., Barreto, S., Giatti, L., Castro, I., ... Crespo, C. (2010). Prevalência de fatores de risco e proteção de doenças crônicas não transmissíveis em adolescentes: resultados da Pesquisa Nacional de Saúde do Escolar (PeNSE), Brasil, 2009. Ciência \& Saúde Coletiva, 15(Supl 2), 3009-3019.

McCarty, C., Ebel, B., Garrison, M., DiGiuseppe, D., Christakis, D., \& Rivara, F. (2004). Continuity of binge and harmful drinking from late adolescence to early adulthood. Pediatrics, 114(3), 714-719.

Miller, C., Strathdee, S., Kerr, T., Li, K., \& Wood, E. (2006). Factors associated with early adolescent initiation into injection drug use: Implications for intervention programs. Journal of Adolescent Health, 38(4), 462-464.

Moller, M., \& Haustein, S. (2014). Peer inuence on speeding behaviour among male drivers aged 18 and 28. Accident Analysis and Prevention, 64(1), 92-99.

Moral, M. V., Rodríguez, F. J., \& Ovejero, A. (2010). Correlatos psicosociales del consumo de sustancias psicoactivas en adolescentes españoles. Salud Pública de México, 52(5), 406-415.

Nkansah-Amankra, S., Diedhiou, A., Agbanu, S. K., Agbanu, H. L., Opoku-Adomako, N. S., \& TwumasiAnkrah, P. (2012). A longitudinal evaluation of religiosity and psychosocial determinants of suicidal behaviors among a population-based sample in the United States. Journal of Affective Disorders, 139(1), 40-51.

Oliveira, H., Martins, L., Reato, L., \& Akerman, M. (2010). Fatores de risco para o uso do tabaco em adolescentes de duas escolas do município de Santo André, São Paulo. Revista Paulista de Pediatria, 28(2), 200-207.

Raffaelli, M., \& Crockett, L. (2003). Sexual risk taking in adolescence: The role of self-regulation and attraction to risk. Developmental Psychology, 39(6), 1036-1046.

Raphaelli, C. O., Azevedo, M. R., \& Hallal, P. C. (2011). Associação entre comportamentos de risco à saúde de pais e adolescentes em escolares de zona rural de um município do Sul do Brasil. Cadernos de Saúde Pública, 27(12), 2429-2440. 
Ritakallio, M., Kaltiala-Heino, R., Kivivuori, J., \& Rimpela, M. (2005). Brief report: Delinquent behaviour and depression in middle adolescence: A Finnish community sample. Journal of Adolescence, 28(1), 155-159.

Roche, K. M., Ahmed, S., \& Blum, R. W. (2008). Enduring consequences of parenting for risk behaviors from adolescence into early adulthood. Social Science \& Medicine, 66(9), 2023-2034.

Sena, C. A., \& Colares, V. (2008). Comportamentos de risco para a saúde entre adolescentes em conflito com a lei. Cadernos de Saúde Pública, 24(10), 2314-2322.

Senna, S. R., \& Dessen, M. A. (2012). Contribuições das teorias do desenvolvimento humano para a concepção contemporânea da adolescência. Psicologia: Teoria e Pesquisa, 28(1), 101-108.

Smith, A., Chein, J., \& Steinberg, L. (2013). Impact of socio-emotional context, brain development, and pubertal maturation on adolescent risk-taking. Hormones and Behavior, 64(2), 323-332.

Steinberg, L. (2004). Risk taking in adolescence: What changes, and why? Annals New Tork Academy of Sciences, 1021(1), 51-58.

Tavares, B., Béria, J., \& Lima, M. (2001). Prevalência do uso de drogas e desempenho escolar entre adolescentes. Revista de Saúde Pública, 35(2), 150-158.

Zhang, X., Wang, H., Xia, Y., Liu, X., \& Jung, E. (2012). Stress, coping and suicide ideation in Chinese college students. Journal of Adolescence, 35(3), 683-690.

World Health Organization. (2004). Sexually transmitted infections in adolescence. Retrieved March 5, 2013, from http://apps.who.int/iris/handle/10665/42902

Recebido: outubro 4, 2013

Versão final: abril 25, 2014

Aprovado: julho 11, 2014 